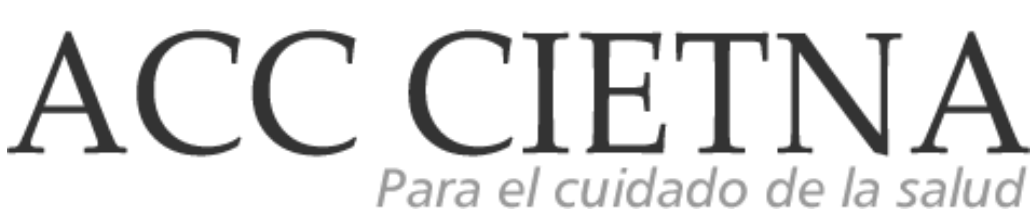

https://doi.org/10.35383/cietna.v8i2.687

e-ISSN: 2309-8570

Universidad Católica Santo Toribio de Mogrovejo

\title{
La etnografía: importancia, relevancia y aportaciones para el cuidado de enfermería
}

\section{Ethnography: importance, relevance and contributions to nursing care}

\author{
Antonieta de Jesús Banda-Pérez ${ }^{*}, 1$, a, b \\ antonieta.banda@uaslp.mx \\ https://orcid.org/0000-0001-9586-0641
}

Reyna Isabel Hernández-Pedroza 2, b

reyna.hernandez@unison.mx

https://orcid.org/0000-0003-0831-4357

Dioselina Negrete-Villafañe ${ }^{3, c, d}$
negretev-dioselina@javeriana.edu.co
https://orcid.org/0000-0002-8626-1863

Andrea Marcela Caviedes-Gil ${ }^{3,} \mathrm{c}, \mathrm{e}$

andreacaviedes@javeriana.edu.co

https://orcid.org/0000-0003-3746-731X

\section{* Autor corresponsal}

\author{
1 Facultad de Enfermería y Nutrición, Universidad \\ Autónoma de San Luis Potosí, México \\ 2 Departamento de Enfermería, Universidad de Sonora, \\ México \\ ${ }^{3}$ Pontificia Universidad Javeriana, Bogotá, Colombia \\ a Doctora en Ciencias de Enfermería \\ b Magister en Ciencias de Enfermería \\ c Licenciada en Enfermería \\ d Enfermera Asistencial de cuidados domiciliarios \\ e Enfermera Asistencial en Unidad de Cuidados \\ Intensivos Neonatales
}

Fechas importantes

Recibido: 2021-06-29

Aceptado: 2021-11-15

Publicado online: 2021-12-17

\section{Resumen}

El presente ensayo corresponde a una revisión sobre la etnografía y su relación con el cuidado de enfermería. En el caso de etnografía, epistemológicamente se trata de sumergir una cultura y el arte de ver, aprender e interpretar la realidad a través del compromiso con los participantes, ya sea de forma abierta o secreta en su entorno. Por lo tanto, el papel de los investigadores en la recopilación de datos es crucial para realizar una investigación etnográfica, ya que este diseño generará datos ricos y profundos, el propósito es comprender los antecedentes o fenómenos en estudio y requiere que el investigador realice visitas de campo y sea reflexivo y considere sus propios supuestos y premisas para fortalecer los resultados de la investigación. Como mencionó Leiniger, la etnoenfermería es considerada como un método de investigación cualitativa que se enfoca en registrar, describir, explicar e interpretar las visiones, significados de los objetos de información de una manera natural, abierta e inductiva, símbolos y vivencias. En relación al contexto de la pandemia COVID-19, se han propuesto nuevas ideas para investigadores que están utilizando métodos etnográficos y diseñando y desarrollando proyectos. Los mismos requieren que se reinventen y ajusten sus estrategias de recolección de datos, para poder llevar a cabo estrategias novedosas para su implementación. Se concluye que existen diferentes mecanismos para desarrollar la etnografía en el cuidado de enfermería e implementar este método junto con diferentes comunidades para superar los diversos desafíos que se enfrentan actualmente, y se ha 
convertido en la máxima prioridad de las necesidades y requerimientos de una sociedad cultural cada vez más diversa.

Palabras clave: Cuidado de enfermería, Etnografía, Etnoenfermería, Investigación en enfermería, COVID-19

\begin{abstract}
This essay is a review of ethnography and its relationship to nursing care. In the case of ethnography, epistemologically it is about immersing a culture and the art of seeing, learning and interpreting reality in through engagement with participants, either openly or secretly in their environment. Therefore, the role of researchers in data collection is crucial in conducting ethnographic research, as this design will generate rich and deep data. The purpose is to understand the background or phenomena under study and require the researcher to conduct field visits, be reflective and consider their own assumptions and premises to strengthen the research findings. As mentioned by Leiniger, ethno-nursing is considered a qualitative research method that focuses on recording, describing, explaining and interpreting the visions, meanings of the objects of information in a natural, open and inductive way, symbols and experiences. In relation to the context of the COVID-19 pandemic, new ideas have been proposed for researchers who are using ethnographic methods and designing and developing projects. These require reinventing and adjusting their data collection strategies in order to carry out new strategies for implementation. It is concluded that there are different mechanisms to develop ethnography to nursing care and implement this method together with different communities to overcome the various challenges they are currently facing, and it has become the top priority of the needs and requirements of an increasingly diverse cultural society.
\end{abstract}

Keywords: Nursing care, Ethnography, Ethno-nursing, Nursing research, COVID-19

\title{
Introducción
}

La investigación cualitativa es un grupo de metodologías, cada una de las cuales ofrece una lente diferente a través de la cual explorar, comprender, interpretar o explicar fenómenos en contextos y entornos reales. En el caso de la etnografía, epistemológicamente se trata de la inmersión en una cultura y el arte de ver, aprender e interpretar la realidad mediante el compromiso con los participantes, ya sea de forma abierta o encubierta en su entorno natural ${ }^{1}$.

Para iniciar este análisis es prudente considerar que la etnografía estudia los diferentes componentes culturales de las personas en su entorno: su relación con el grupo, sus creencias, sus símbolos y rituales, los objetos que utilizan, sus costumbres y valores, etc ${ }^{2}$.

Además, cabe destacar que la etnografía entre sus principales características es la completitud y el contexto, que implica colocar las observaciones etnográficas en una perspectiva amplia, lo que significa que el comportamiento de las personas solo puede entenderse en su propio contexto, es por ello por lo que la etnografía se compone de materiales cotidianos, por lo que el trabajo de campo a largo plazo ayudará a los investigadores a comprender el significado de la vida cotidiana de las personas.

Es por ello por lo que la etnografía está siempre en un entorno natural o "campo", por lo que los investigadores pueden profundizar más directamente la vida de los miembros de grupos culturales1. Los investigadores deben permitir que los participantes culturales hablen y descubran cómo construyen su propio mundo de experiencia; cómo y desde qué categoría generar arreglos; el fenómeno sociocultural en sus mentes; ¿qué significa este atributo para ellos?

Por consiguiente, el papel del investigador en la recopilación de datos es primordial para llevar a cabo un estudio etnográfico, ya que este diseño produce datos ricos y profundos con el objetivo 
de dar sentido al contexto o a los fenómenos investigados, y requieren que el investigador sea reflexivo al realizar el trabajo de campo, dando cuenta de sus propios supuestos y presuposiciones para fortalecer los hallazgos.

Por otra parte, tenemos en la profesión de Enfermería el método de investigación de etnoenfermería que fue desarrollado para analizar los fenómenos culturales y asistenciales detectados. Como lo refiere Leininger la etnoenfermería se trata como un método de investigación cualitativa, que se enfoca en registrar, describir, explicar e interpretar los puntos de vista, significados, símbolos y experiencias de vida de los objetos de información de una manera naturalista, abierta e inductiva. Leininger fue una de las primeras enfermeras que publico métodos de investigación cualitativos en los primeros años de la década de $1960^{3}$.

De tal suerte, que la investigación etnográfica ayuda a las ciencias de la salud, especialmente a la enfermería, porque analiza la cultura de un grupo de personas para comprender la visión de los informantes del mundo en la que los investigadores describen y explican patrones, valores, comportamientos, creencias y lenguaje compartiendo y aprendiendo por miembros de grupos culturales específicos.

Por otra parte, en el contexto de la pandemia COVID-19 se presentaron nuevos escenarios para los investigadores que desarrollaban proyectos con enfoques y diseños cualitativos, retándolos a reinventarse y adecuar sus estrategias para la recolección de los datos, por ello es importante no dejar de lado esta situación para reflexionar.

Podemos condensar lo dicho hasta aquí, con el hecho de que el presente ensayo tiene como propósito u objetivo reflexionar sobre la importancia, relevancia y aportaciones de la etnografía para el cuidado de enfermería. Se integra de 4 temas generales entorno a los antecedentes de la etnografía, aportaciones de la etnografía al campo de la enfermería, trabajo de campo con las comunidades indígenas, técnicas cualitativas abordadas durante la pandemia COVID-19 y experiencias de estudios sobre etnoenfermería.

\section{Desarrollo}

\section{Antecedentes de la etnografía}

¿Es la etnografía una técnica de investigación aplicable en muchas ciencias?

A continuación, retomaremos un poco la historia de la etnografía, ya que mencionando estos antecedentes nos damos cuenta de las diferentes ciencias que han utilizado la etnografía para sus investigaciones a través del tiempo.

En primer lugar, el método etnográfico de estudiar los grupos humanos comenzó con los antropólogos británicos, que desarrollaron una forma de investigación etnográfica centrada en la antropología social entre los siglos XIX y XX. Los representantes más influyentes de estas escuelas son AR Radcliffe-Brown y Bronislaw Malinowski ${ }^{4}$.

Después los antropólogos estadounidenses se interesaron por estudiar las culturas de los pueblos nativos americanos, cuyas formas tradicionales de vida en ese momento ya habían tenido cambios, de esta manera la antropología de los estados unidos llego a denominarse antropología cultural pues reconstruyeron la memoria histórica de los supervivientes, el antropólogo cultural más influyente fue Franz Boas ${ }^{4}$. 
Luego los sociólogos de la universidad de Chicago desde 1920 se acogieron a los métodos de investigación de campo etnográfico de los antropólogos, para aplicarlos en los grupos sociales comunidades estadounidenses más modernas.

La escuela de Chicago influenció a varios campos como la educación, los negocios, la salud pública, la enfermería, trabajadores sociales y las comunicaciones ${ }^{4}$.

En primer lugar, resaltamos a la enfermera Madeleine Leinninger fundadora de la enfermería transcultural, durante su estudio doctoral se introdujo durante dos años en una cultura indígena de Nueva Guinea donde tuvo la oportunidad de convivir con ellos y aprender de su cultura, creencias y mitos ${ }^{5}$.

Finalmente, estudios en profundidad, acompañados de sus experiencias directas, alentaron al desarrollo continuo de su teoría de los cuidados culturales y el método de etnoenfermería. Sus investigaciones y desarrollos teóricos han ayudado desde entonces a los profesionales de enfermería a comprender las diferencias culturales que se otorgan en la atención a la salud y la enfermedad ${ }^{5}$.

Para concluir la etnografía no solo se limita a estudiar las comunidades indígenas, a nivel de enfermería la podemos utilizar al hacer investigaciones en el ámbito hospitalario, domiciliario y universitario.

Desde el comienzo de la historia de la etnografía hay diferentes disciplinas que la vienen utilizando para adelantar sus estudios y cómo podemos observar la enfermería no se ha quedad atrás y cobró su propia esencia etnográfica a través de la etnoenfermeria.

\section{Aportaciones de la etnografía al campo de la enfermería}

¿Es la etnografía base y principio del cuidado transcultural de enfermería?

Cuidar es un arte, y en la sociedad actual no es tarea fácil, pues implica responder a las necesidades particulares e individuales del ser humano su familia y su entorno; la necesidad de cuidar ha sido siempre universal y es base y esencia del profesional de enfermería, pues enfermería mucho más que una carrera, es una ciencia en la que se enlazan el conocimiento, la razón y el corazón, de igual forma la constante evolución, implementación de bases científicas y la construcción de pensamiento crítico ha permitido que a través del tiempo se cualifique e identifique como una profesión independiente, totalmente humana y al servicio de los demás ${ }^{6}$.

Las personas son seres psicosociales biológicas, son inseparables del entorno cultural y estructural, el estilo de vida y la visión del mundo. Por tanto, es difícil imaginar que elementos culturales como creencias religiosas, valores, costumbres, reglas familiares, mitos y tabúes no interfieran con los procesos naturales de salud y enfermedad.

Así como el concepto de salud, la explicación de los síntomas, los cuidados y tratamientos que promueven y restablecen la salud están completamente definidos por la cultura a la que pertenecen, esto obliga a que, si se quiere explorar parcialmente un campo desconocido, su exploración es más empática en su intervención, precisa, concisa y delicada.

Teniendo en cuenta que la cultura es eje fundamental en el curso natural de la enfermedad, la etnografía es una herramienta de trabajo indispensable para enfermería 
pues permite el abordaje de situaciones de cuidado que permiten entender y comprender los comportamientos y las acciones de cuidado que utilizan los pacientes o sujetos de estudio.

Sus aportes son múltiples a la disciplina y son fundamentales a la hora de identificar el cuidado transcultural de enfermería pues permite establecer conocimientos relacionados a la comprensión de las conductas de salud de las diferentes sociedades multiculturales que existen , apoya la naturaleza epistemológica en su relación cotidiana con los grupos humanos, esto permite a enfermería profundizar en la cultura estilo de vida procesos de salud que se quiere descubrir, para facilitar las intervenciones de cuidado que requieren las diferentes comunidades ${ }^{7}$.

Adicional a esto, el contacto directo y cotidiano que establecen los enfermeros con sus pacientes da un plus fundamental para desarrollar la etnografía pues gracias a este seguimiento continuo y la oportunidad de descubrir diferentes aspectos de la vida diaria, permiten identificar el vacío existente para que el profesional de enfermería fundamente su cuidado con base en lo que las personas transmiten.

Así mismo Madeleine Leininger desde la creación de su teoría transmite una aproximación integral al conocimiento de los cuidados, integrando los fundamentos de enfermería con las bases y principios de la ciencia social, y la antropología. Leininger expresa que es de gran importancia dar a conocer una enfermería culturalmente competente, sustentada en un modelo teórico, que permita al personal de enfermería ampliar su conocimiento y comprensión de las diferentes culturas ${ }^{8}$, adaptando la etnografía al estudio del objeto específico de la enfermería: las personas, su entorno y su familia.

La etnografía es una práctica enriquecedora para la disciplina pues cumple un papel fundamental, el de dar a conocer una experiencia cultural, con la finalidad de identificar elementos propios de cada grupo poblacional relacionados con la salud y sobre todo para determinar los cuidados culturales en las áreas de la salud pública, que permiten describir y descubrir los patrones formativos de un grupo con relación a la salud y prácticas de cuidado cultural, las creencias de tipo cultural, los comportamientos culturales que pueden ser determinantes junto con los factores de la estructura social en los resultados en la salud de una sociedad determinada y contribuye desde otro ángulo a la enfermería basada en evidencia, y es cuando vemos un claro ejemplo de esta esencia etnográfica o de etnoenfermeria, por tal razón se quiso enlazar la experiencia de un trabajo de campo en comunidades indígenas.

\section{Trabajo de campo en comunidades indígenas}

¿Sera importante el poder comunicarnos con los participantes del estudio a través de su lengua nativa? la lengua materna de una comunidad es el elemento básico para estudiar dentro del trabajo de campo cualitativo. Ya que cuando el investigador y el participante pueden comunicarse cara a cara en el mismo idioma, compartir el mismo idioma y no hay diferencia entre los dos, la relación se vuelve más estrecha. Por tal motivo, el idioma es la perspectiva de la cultura y es la mejor manera de comprender la cultura de la otra parte.

El desconocimiento del idioma representa un problema difícil, lo que lleva a algunos investigadores a aprender el idioma, o de lo contrario, buscan la ayuda de un traductor en la comunidad. Esto ha llevado a poner en dilema si existe algún problema en la 
interpretación o expresar tal cual lo dijo el participante ya que con un traductor se puede correr el riesgo de realizar descripciones e interpretaciones sin la profundidad como fue expresada.

Desafortunadamente, no siempre es posible aprender el idioma de la comunidad donde se está trabajando, lo que puede algunas veces dificultar la comunicación o hacerla menos fluida. Siempre existe la posibilidad de encontrar un buen traductor que especifique tal como lo expreso la participante y más si el investigador puede entablar una buena relación con la comunidad se abre ese espacio donde se posibilita tener una buena interpretación del traductor. Es por esto que además de poder entablar una adecuada comunicación con nuestros participantes es importante la observación, ya que los investigadores muchas veces se convierten en partícipes de escenarios culturales, es decir, la observación participante no es solo una técnica simple, sino que se considera la base de la investigación etnográfica porque a partir de esto podemos relacionarnos con el grupo de actores sociales así como de su cultura, creencias, símbolos, rituales, costumbres, valores, entre otros.; y es así como de alguna manera hacen del investigador parte del fenómeno de estudio ${ }^{9}$.

¿Por qué es importante la presencia del investigador en el trabajo de campo?

Es importante la presencia del investigador en todo el trabajo de campo ya que es fundamental, porque el estudio de la cultura requiere un procesamiento en profundidad con participantes que son parte de esa cultura. La principal forma en que los investigadores se convierten en herramientas es a través de entrevistas, observaciones, formando parte de un grupo cultural seleccionado para revelar fenómenos de investigación, así como registrando datos culturales y comprobando reliquias culturales ${ }^{10}$.

Uno de los aspectos más destacados es identificarse con los líderes comunitarios y ganar confianza a través de la ropa. Poder vestirse como parte de la misma comunidad con la que los investigadores interactuarán es el primer paso para ganar confianza. Es por esto que además de la observación, los investigadores a menudo se convierten en participantes del entorno cultural, es decir, participar en la observación no es solo una técnica simple, se considera la base de la investigación etnográfica. Por ello se relaciona con el grupo de participantes, su cultura, sus creencias, símbolos, rituales, los objetos que utilizan, sus costumbres, valores, etc....; este es un método de triangulación de observaciones y entrevistas y su uso. Hacer que los investigadores formen parte del fenómeno de la investigación. Ser aceptado por el grupo es importante porque brinda confianza y apertura inmediatas a los participantes del estudio?.

Poder interactuar y ser parte del fenómeno de aprendizaje que se requiere develar es algo que nunca se olvida, porque, aunque se pueda encontrar con mal tiempo, (ya sea lluvia, calor, frio exagerada) así que se debe persevera a pesar de lo que se pueda presentar con el clima. Además, el poder explicar la cultura de una comunidad es la verdadera esencia de la investigación etnográfica. La etnografía es el único método de investigación cuyo único propósito es comprender el estilo de vida de los individuos conectados a través de una cultura es por ello que Los investigadores etnográficos deben trabajar duro para descubrir y explicar el significado cultural que se encuentra en un grupo conectado ${ }^{10}$.

Es de vital importancia resaltar que los investigadores deben tener algunas características básicas, como sensibilidad e imparcialidad, fuerte capacidad de observación, buena comunicación para generar confianza y fácil adaptación ante la presencia de factores 
inestables. Estar abierto a la novedad, la diferencia y la diversidad para que los datos se puedan recopilar, describir e interpretar sin estereotipos. Es flexible y no inoportuno, capaz de observar a los actores sociales o interactuar con ellos e involucrarse de sus costumbres, valores, creencias y prácticas es decir aprender con ellos ${ }^{11,12}$. Hay que mencionar, además que la etnoenfermería indaga los factores de la estructura social, cultural, la educación, los ingresos económicos, valores, creencias que influye en las experiencias de los participantes de estudio, según su percepción cultural o conocimiento subjetivo a través de las características culturales de las personas, manifestadas por el lenguaje, la experiencia, las creencias y los sistemas de valores para expresar información sobre fenómenos enfermeros reales o potenciales, como pueda ser la asistencia, la salud y los factores ambientales, es por ello que debemos dejar de lado el etnocentrismo ya que es una actitud hacia las diferencias culturales, que consiste en creer que la propia cultura es la única adecuada, incluso mejor que cualquier otra cultura ${ }^{13}$.

La experiencia de enfermería en el entorno transcultural explica y requiere la necesidad de seguir investigando y formando profesionales del cuidado de la salud centrados plenamente en el desarrollo de la competencia cultural.

\section{Técnicas cualitativas abordadas durante la pandemia COVID-19 y experiencias de estudios sobre etnoenfermería}

Tras la revisión literaria y conocer un poco más sobre la historia, aportaciones e importancias de la etnografía surge el interrogante sobre ¿qué sucede con la etnografía en la actualidad y a un más en el contexto de la pandemia actual del COVID- 19?

La investigación cualitativa implica el acercarse de manera profunda y personal con los sujetos de la investigación, con el propósito de compartir formas de ver al mundo, y sobre todo asumiendo una actitud empática. La pandemia COVID-19 modificó el contexto en el cual el realizar este involucramiento con las personas sujetos de investigar bajo el enfoque cualitativo tradicional dejó de ser cara a cara, para lograrse a través de medios digitales, redes sociales y llamadas telefónicas.

Es posible que todavía se presente en los investigadores una resistencia el pasar de lo tradicional a un cambio, sobre todo porque fue en un contexto donde el aislamiento social era parte del llamado mundial para la contención del virus SARS-COV-2, de tal manera que fue necesario hacer uso de la creatividad e innovación para el seguimiento de proyectos de investigación que seguramente se inmovilizaron al inicio de la pandemia. Ahora, la cuestión es ¿cómo continuaron los investigadores para preservar la esencia del enfoque cualitativo en época de pandemia, donde el mandato mundial era prácticamente cero contactos personales? y ¿cuáles son las experiencias de enfermería en la investigación cualitativa en el contexto del COVID-19 en el diseño de la etnografía?

A continuación, se pretende brindar unas respuestas a las preguntas anteriores, sin intención de mostrar resultados en base a una búsqueda sistemática u otra metodología cuantitativa y rigurosa, es simplemente a partir de la postura de que aún en contextos de crisis, es posible continuar y es importante no desistir de la investigación cualitativa, ya que con ella podemos conocer el sentir, el pensar y aproximarnos a las experiencias más cercanas y sentidas de las personas en tiempos donde se prohíbe el contacto humano y solo queda convivir en un espacio reducido, en soledad o si bien nos va en familia y lo que conlleva todo ello. 
En cuanto a la pregunta de ¿cómo continuaron los investigadores para preservar la esencia del enfoque cualitativo en época de pandemia, donde el mandato mundial era prácticamente cero contactos personales?, según Hernán, Lineros y Ruiz ${ }^{14}$, durante la pandemia COVID-19, que persiste todavía, se necesitó matizar o reorientar las técnicas cualitativas, naturalistas o etnográficas, es decir las formas de investigar las conductas sociales que brinden luz sobre la relación congruente o la falta de la misma entre práctica social específica y el discurso, con el propósito de llevar a cabo un trabajo de campo útil para comprender un fenómeno determinado. Para ello, fue imprescindible durante el confinamiento aprender o reaprender a trabajar de manera remota y autónoma.

Es aquí, donde autores como Hine ${ }^{15}$ y Sánchez y $\operatorname{Ortiz}^{16}$, refieren la necesidad de situar la etnografía hacia la ciberetnografía, sin perder el enfoque tanto crítico como riguroso. Lo anterior, permite entonces la reflexión sobre la oportunidad que brindó o está brindando COVID-19, para lograr esta nueva forma de aproximarnos a la realidad y experiencia de las personas con un enfoque cualitativo, es así, que las técnicas a utilizar para esta época de confinamiento y aislamiento son la observación, conversación y documentación, donde la modalidad es videoconferencias individuales o grupales, de manera virtual, haciendo uso de las técnicas de entrevistas individuales y/o grupales, a través de una cámara (ordenador, smartphone, vídeo o alternativas) y por medio de plataformas como Skype, Jitsi, Facebook, WhatsApp, Zoom, herramientas de Moodle o Google forms, Kahoot, LimeSurvey, Twitter, entre otros ${ }^{16}$.

Aunado a lo anterior, lo importante es preservar el rigor y las cuestiones éticas, sobre todo por la pandemia, de tal manera que se conserve el respeto, confidencialidad y privacidad, de los participantes. Es entonces, que los investigadores pueden y pudieron continuar con sus proyectos basados en la investigación etnográfica en estos tiempos difíciles para todos.

De tal manera que la respuesta de la siguiente pregunta ¿cuáles son las experiencias de enfermería en la investigación cualitativa en el contexto del COVID-19 en el diseño de la etnografía?, muestra la evidencia de este logro por seguir adelante con los proyectos, específicamente para el caso de la etnoenfermería, la cual, como se describió anteriormente, permite abordar los cuidados culturales de las personas en su propio contexto y realidad, lo cual, es relevante para mejorar las políticas de salud, así como los programas específicos de salud para las problemáticas de cada grupo etario.

Como lo refiere Sánchez et. al. ${ }^{17}$ concluye en su investigación que los profesionales de Enfermería solamente podrán cuidar de manera competente cuando conozcan las particularidades de cada paciente, con el respeto que se merece, indistintamente de su origen, religión o cultura, y aprendiendo de cada uno de ellos. En el caso del estudio de Garay et. al. ${ }^{18}$ a través del método de la etnoenfermería concluyó que en la atención de las mujeres se práctica de manera natural la violencia obstétrica, independientemente del género (masculino o femenino), además de identificar otras categorías emergentes de análisis como la soledad, el androcentrismo y la corporeidad que la etnoenfermería debe considerar para mejorar la atención integral y la calidad en los cuidados brindados a esta población. Así mismo, en otro estudio fue posible identificar los cambios en el funcionamiento familiar, que incluso pueden ser la desintegración de la familia, así como en roles de los cuidadores, los cuales suceden a partir de la experiencia de muerte materna, y por consecuencia, afectando el rendimiento escolar e impactando considerablemente en el área emocional de la familia ${ }^{19}$. 
En el caso de Ojeda et. al. ${ }^{19}$, los resultados arrojaron que se confirmó el aporte de la etnoenfermería, a partir de señalar el valor de la identidad en la comunidad de mapuche en Puerto Aguirre, Chile para preservar el cuidado cultural; la presencia de mujeres como parte esencial para brindar atención a los otros integrantes de la comunidad; además de que los mapuches que otorgan valor a las prácticas de cuidados de los profesionales, así como el hecho de que las utilizan como sus mismas practicas basadas en su cultura.

Lo estudios anteriores nos brindan una pincelada de lo que es la experiencia de investigación con el método etnográfico, el cual enriquece a la disciplina de enfermería, aun cuando falta mucho por explorar, este método permite encontrarse con el cuidado humano de una manera íntima y profunda, como solo se puede lograr en una relación interpersonal y terapéutica: enfermera-paciente.

\section{Conclusiones}

La etnografía es un campo aun poco explorado de la enfermería, pero que día a día y frente a los cambios evolutivos que experimenta la sociedad, brinda los cimientos necesarios y da a conocer la importancia de incorporarla en los diferentes ámbitos en los que se desempeña el profesional, pues permite establecer interrelaciones destinadas a llevar a cabo el proceso de cuidar desde la comprensión de la realidad cultural y la diversidad humana.

Existen diferentes mecanismos que permiten desarrollar la etnografía y llevar a cabo el acercamiento con las diferentes comunidades, superando así los diferentes retos que se enfrentan en la actualidad y logrando estar a vanguardia de las necesidades y requerimientos de una sociedad cada vez más pluricultural. La investigación etnográfica es útil para las ciencias de la salud, especialmente la enfermería, porque analiza la cultura de un grupo de personas para comprender las visiones del mundo de los informantes, donde los investigadores describen y explican patrones, valores, comportamientos, creencias y el intercambio de idiomas y el aprendizaje de los miembros de grupos culturales específicos.

La pandemia COVID-19, cerro fronteras y puertas de la casas, sin embargo, la etnografía y adaptar las técnicas cualitativas tradicionales a un contexto de confinamiento y aislamiento, permitió continuar con la exploración, la aproximación y la profundización de los fenómenos que solo bajo este enfoque cualitativo podría abordarse, ya que la comprensión de las experiencias de cuidados culturales, invitan a individualizar el cuidado humano, y para la práctica de enfermería, es indispensable e invaluable dicho cuidado.

\section{Referencias}

1. Pla M. El rigor en la investigación cualitativa. Aten Primaria [Internet]. 1999 [Consultado $10 \mathrm{Abr}$ 2021]; 24: 295-300. Disponible en: http://www.unidaddocentemfyclaspalmas.org.es/resources/3+Aten+Primaria+1999.El+Rigor+de + la+Investigaci\$C3\$B3n+Cualitiativa.pdf

2. Organización Panamericana de la Salud. Investigación cualitativa en enfermería: contexto y bases conceptuales [Internet]. Washington, D. C.: OPS; 2008 [Consultado 10 Abr 2021]. Disponible en: https://iris.paho.org/handle/10665.2/51581

3. Maira $M$, Palomare $V$, del Barrio $N$, Atares $A$, Piqueras $M$, Miñez $T$. Madeleine Leininger, articulo monográfico. Revista Sanitaria de Investigación (RSI): 2021 [Consultado 10 Abr 2021]. Disponible en: https://www.revistasanitariadeinvestigacion.com/madeleine-leininger-articulo-monografico/

4. Angrosino, M. Etnografía y observación participante en investigación cualitativa. Madrid: Ediciones Morata 2012. 143p. 
5. Raile Alligood M, Tomey AM. Modelos y teorías en enfermería. Séptima edición. Elsevier 2011.797p.

6. Lagoueyte Gómez MI. El cuidado de enfermería a los grupos humanos. Rev Univ Ind Santander Salud. 2015; 47(2):209-213

7. Organización Panamericana de la Salud. Brasil, Universidad de Federal de Santa Catarina. Investigación cualitativa en enfermería. Metodología y didáctica [Internet]. Washington, DC: OPS; 2013 [Consultado 10 Abr 2021]. Disponible en: https://iris.paho.org/handle/10665.2/51587

8. Guba EG, Lincoln YS. Effective evaluation: improving the usefulness of valuation result through responsive and naturalistic approaches. American Journal of Evaluation [Internet] 1981 [Consultado 13 Abr 2021]; 3(4): 17-22. Disponible en: http://journals.sagepub.com/doi/abs/10.1177/109821408200300406\#articleCitationDownloadCon tainer

9. Montes L. Una ventana epistémica a la (inter) subjetividad: las potencialidades del método etnográfico. Social Science [Internet]. 2016 [Consultado 15 Abr 2021]; 17(1): 1-20. Disponible en: https://www.ssoar.info/ssoar/handle/document/50623\#

10. Roper JM, Shapira J. Ethnography in nursing research. Thousand Oaks: SAGE Publications [Internet]. 2000 [Consultado 15 Abr 2021]. 150 p. Disponible en: https://onlinelibrary.wiley.com/doi/epdf/10.1046/j.1466-7657.2000.00052.x

11. Do Prado M, de Souza M, Carroro T. Investigación cualitativa en enfermería, contextos y bases contextuales. Organización Panamericana de la Salud [OPS]: Washington, D.C.2008. 233p.

12. Leinninger M. Teoría de la diversidad y universalidad de los cuidados culturales. En: Raile M. Modelos y teorías en enfermería. Octava edición. Editorial: Elsevier. Barcelona: España; 2015. $728 \mathrm{p}$.

13. Hernán, M., Lineros-González, C., Ruiz-Azarola, A. Cómo adaptar una investigación cualitativa a contextos de confinamiento. Gaceta Sanitaria. 2020; 35. 10.1016/j.gaceta.2020.06.007.

14. Hine C. Etnografía virtual. Barcelona: UOC; 2004. p. 218. Disponible en: https://www.uoc.edu/dt/esp/hine0604/hine0604.pdf.

15. Sánchez W, Ortiz P. La netnografía, un modelo etnográfico en la era digital. Espacios. 2017; 38: 1-14. Disponible

en: https://doi.org/http://www.revistaespacios.com/a17v38n13/a17v38n13p28.pdf.

16. Sánchez M, Segura A, Gallardo M, Alemany I. Enfermería Transcultural. Formación de los futuros profesionales de Enfermería en España. Index de Enfermería [Internet]. 2018 [Consultado el 6 de mayo de 2021]; 27(4): 247-250. Disponible en: http://scielo.isciii.es/scielo.php?script=sci_arttext\&pid=S1132-12962018000300015

17. Garay J., Jiménez, V., Santos, M., Félix, M. Violencia obstétrica: una mirada desde el interaccionismo simbólico y la etnoenfermería. 2017. Disponible en: http://enfermeria2017.sld.cu/index.php/enfermeria/2017/paper/viewPaper/420

18. Romero-Guzmán, I., Muñoz-Monteroza, D., Benitez-Cheij, L. Experiencia familiar frente a la muerte materna. Revista ciencia y cuidado, 2020; 17 (2). Disponible en: https://dialnet.unirioja.es/servlet/articulo?codigo $=7490964$

19. Ojeda, M., Heidemann, I., Schulter, T., Villa, S. etnoenfermería: cuidados culturales en una comunidad mapuche en Puerto Aguirre, región de aysén-chile. Texto \& Contexto - Enfermagem [Internet]; 29(spe): e20190262. Disponible en: https://doi.org/10.1590/1980-265x-tce-2019-0262 


\section{Editor}

Escuela de Enfermería de la Universidad Católica Santo Toribio de Mogrovejo, Chiclayo, Perú

\section{Cómo citar este trabajo}

Banda-Pérez A, Hernández-Pedroza R, Negrete-Villafañe D, Caviedes-Gil A. La etnografía: importancia, relevancia y aportaciones para el cuidado de enfermería. Acc Cietna: para el cuidado de la salud [Internet]. 2021; 8(2): 106 - 116. Disponible en: https://doi.org/10.35383/cietna.v8i2.687

\section{Financiación}

El presente artículo no cuenta con financiación específica de agencias de financiamiento en los sectores público o privado para su desarrollo y/o publicación.

\section{Conflicto de interés}

Los autores del artículo declaran no tener ningún conflicto de intereses en su realización.

(C) Los autores. Este artículo es publicado por la Revista Acc Cietna: para el cuidado de la salud de la Escuela de Enfermería, Universidad Católica Santo Toribio de Mogrovejo. Este es un artículo de acceso abierto, distribuido bajo los términos de la Licencia Creative Commons Atribución-NoComercial-CompartirIgual 4.0 Internacional (CC BY-NC-SA 4.0), que permite el uso no comercial, distribución y reproducción en cualquier medio, siempre que la obra original sea debidamente citada. 